\title{
Convexity-preserving properties of set-valued ratios of affine functions
}

\author{
Alexandru Orzan and Nicolae Popovici
}

\begin{abstract}
The aim of this paper is to introduce some classes of set-valued functions that preserve the convexity of sets by direct and inverse images. In particular, we show that the so-called set-valued ratios of affine functions represent such a class. To this aim, we characterize them in terms of vector-valued selections that are ratios of affine functions in the classical sense of Rothblum.
\end{abstract}

Mathematics Subject Classification (2010): 54C60, $26 \mathrm{~B} 25$.

Keywords: Set-valued affine function, single-valued selection, ratio of affine functions, generalized convexity.

\section{Introduction}

Various classes of fractional type real-valued or vector-valued functions have been introduced in the literature, being nowadays well recognized for their important applications in scalar and vector optimization (see, e.g., Avriel et al. [2], Cambini and Martein [4], Göpfert et al. [6], Stancu-Minasian [13], and the references therein).

According to Rothblum [11], a vector-valued function $f: D \rightarrow \mathbb{R}^{m}$, defined on a nonempty convex set $D \subseteq \mathbb{R}^{n}$, is said to be a ratio of affine functions if there exist a vector-valued affine function $g: \mathbb{R}^{n} \rightarrow \mathbb{R}^{m}$ and a real-valued affine function $h: \mathbb{R}^{n} \rightarrow \mathbb{R}$, such that

$$
D \subseteq\left\{x \in \mathbb{R}^{n} \mid h(x)>0\right\}
$$

and

$$
f(x)=\frac{g(x)}{h(x)}, \forall x \in D .
$$

These functions are known to preserve the convexity of sets by direct and inverse images. Moreover, they transform the line segments in line segments (possibly degenerated into singletons). This concept along with the above mentioned convexitypreserving properties can be naturally extended for vector-valued functions acting between general real linear spaces. 
Recently, Orzan [10] investigated a class of set-valued ratios of affine functions, defined similarly to (1.1), by replacing $g$ with a set-valued function that is affine in the sense of Tan [14]. As shown in [10], these functions preserve the convexity of sets by direct images. A natural question is whether they preserve the convexity of sets by inverse images too. The aim of this paper is not only to give a positive answer to this question, but also to identify some broader classes of set-valued functions that preserve the convexity of sets by direct and inverse images.

We start by recalling some basic definitions of set-valued and convex analysis in Section 2. The concept of set-valued affine function, as defined by Tan [14], is investigated in Section 3. In particular, we show that the inverse of such a function is affine as well (in contrast to other concepts of affine set-valued functions, cf. Kuroiwa et al. [7, Ex. 2]). Section 4 is devoted to the ratios of affine functions. In Section 4.1 we briefly present how the classical results of Rothblum [11] can be extended from finite-dimensional Euclidean spaces to general linear spaces. Subsection 4.2 is devoted to our main results. First we introduce the class of set-valued ratios of affine functions, by refining the definition proposed in [10]. Theorem 4.8 gives an explicit representation of these functions, which plays a key role in establishing the convexitypreserving properties of the set-valued ratios of affine functions. Moreover, Theorems 4.10 and 4.11 show that these properties are still valid in some broader classes of set-valued functions. We conclude the paper by rising an interesting open question in Section 5.

\section{Preliminaries}

Throughout this paper we assume that $X$ and $Y$ are two real linear spaces. As usual in set-valued analysis (see, e.g., Aubin and Frankowska [1]), for any set-valued function $F: X \rightarrow \mathcal{P}(Y)$ we denote by

$$
\operatorname{dom} F=\{x \in X \mid F(x) \neq \emptyset\}
$$

the domain of $F$. We say that $F$ is proper if $\operatorname{dom} F \neq \emptyset$. The (direct) image of a set $A \subseteq X$ by $F$ is defined as

$$
F(A)=\bigcup_{x \in A} F(x)
$$

There are different manners to define the inverse image of a set $B \subseteq Y$ by a set-valued map $F$, two of them being currently used in set-valued analysis [1], namely:

$$
\begin{aligned}
& F^{-1}(B)=\{x \in X \mid F(x) \cap B \neq \emptyset\}, \\
& F^{+1}(B)=\{x \in X \mid F(x) \subseteq B\} .
\end{aligned}
$$

The set $F^{-1}(B)$ is called the inverse image of $B$ by $F$ and $F^{+1}(B)$ is called the core of $B$ by $F$ (also known as the lower inverse image and the upper inverse image of $B$ by $F$, respectively). They are related by

$$
F^{+1}(B)=X \backslash F^{-1}(Y \backslash B) .
$$


Remark 2.1. Let $F: X \rightarrow \mathcal{P}(Y)$ be a set-valued function. Given a set $B \subseteq Y$ we have

$$
\begin{aligned}
& F^{-1}(B) \subseteq \operatorname{dom} F, \\
& F^{+1}(B)=\{x \in \operatorname{dom} F \mid F(x) \subseteq B\} \cup(X \backslash \operatorname{dom} F) .
\end{aligned}
$$

Notice that, instead of (2.2), Berge [3] defined a slightly different concept of upper inverse image of $B$, namely $\{x \in \operatorname{dom} F \mid F(x) \subseteq B\}$, which actually means $F^{+1}(B) \cap \operatorname{dom} F$.

Remark 2.2. Let $F: X \rightarrow \mathcal{P}(Y)$ be a set-valued function. Then, for all sets $A \subseteq X$ and $B \subseteq Y$, the following equivalence holds:

$$
A \subseteq F^{+1}(B) \Longleftrightarrow F(A) \subseteq B .
$$

Remark 2.3. According to [1], the inverse of $F$ is the set-valued function $F^{-1}: Y \rightarrow$ $\mathcal{P}(X)$ defined for any $y \in Y$ by

$$
F^{-1}(y):=\{x \in X \mid y \in F(x)\} .
$$

Notice that $\operatorname{dom} F^{-1}=F(X)$ and $F^{-1}(Y)=\operatorname{dom} F$. Also, for every set $B \subseteq Y$, we have

$$
F^{-1}(B)=\bigcup_{y \in B} F^{-1}(y)=\{x \in X \mid F(x) \cap B \neq \emptyset\} .
$$

It is important to notice that, according to (2.1) and (2.6), one can use without any confusion the same notation, $F^{-1}(B)$, for both the lower inverse image of $B$ by $F$ and the direct image of $B$ by $F^{-1}$.

Remark 2.4. Every vector-valued function $f: D \rightarrow Y$ defined on a nonempty set $D \subseteq X$ can be identified with a set-valued function $F: X \rightarrow \mathcal{P}(Y)$,

$$
F(x)=\left\{\begin{array}{cl}
\{f(x)\} & \text { if } x \in D \\
\emptyset & \text { if } x \in X \backslash D .
\end{array}\right.
$$

Obviously, $\operatorname{dom} F=D$ and, for all $A \subseteq X$ and $B \subseteq Y$, we have

$$
\begin{aligned}
F(A) & =f(A \cap D):=\{f(x) \mid x \in A \cap D\}, \\
F^{-1}(B) & =f^{-1}(B):=\{x \in D \mid f(x) \in B\}, \\
F^{+1}(B) & =f^{-1}(B) \cup(X \backslash D), \text { i.e., } F^{+1}(B) \cap D=f^{-1}(B) .
\end{aligned}
$$

In particular, the second relation shows that one can define the set-valued function $f^{-1}: Y \rightarrow \mathcal{P}(X)$ as $f^{-1}(y):=F^{-1}(y)$ for all $y \in Y$.

The aim of this paper is to study some classes of set-valued functions that preserve the convexity of sets by direct and inverse images. Therefore we will adopt the following conventional notations in a real linear space $V$ (in particular, $X$ or $Y$ ). Given $S, S^{\prime} \subseteq V, \lambda \in \mathbb{R}$ and $v_{0} \in V$, we set:

$$
\begin{aligned}
& S+S^{\prime}:=\left\{v+v^{\prime} \mid\left(v, v^{\prime}\right) \in S \times S^{\prime}\right\}, \quad v_{0}+S:=\left\{v_{0}\right\}+S, \\
& \lambda S:=\{\lambda v \mid v \in S\}, \text { and } \frac{S}{\lambda}:=\frac{1}{\lambda} S \text { whenever } \lambda \neq 0 .
\end{aligned}
$$


Recall that a set $S \subseteq V$ is called convex if $(1-t) S+t S \subseteq S$ for all $t \in[0,1]$. The convex hull of a set $S$, i.e., the smallest convex subset of $V$ that contains $S$, will be denoted by conv $S$. For convenience, when the convex hull applies only to the first term of a sum of two sets we will simply write conv $S+S^{\prime}$ instead of $(\operatorname{conv} S)+S^{\prime}$. A set $S \subseteq X$ is called affine if $(1-t) S+t S \subseteq S$ for all $t \in \mathbb{R}$. If $S$ is nonempty, then $S$ is affine if and only if there exists a (unique) linear subspace $L$ of $V$ such that $S=v+L$ for all $v \in S$.

\section{Affine set-valued functions}

Recall that a vector-valued function, $a: E \rightarrow Y$, defined on a nonempty affine set $E \subseteq X$, is said to be affine if for any $x^{1}, x^{2} \in E$ and $t \in \mathbb{R}$ we have

$$
a\left((1-t) x^{1}+t x^{2}\right)=(1-t) a\left(x^{1}\right)+t a\left(x^{2}\right) .
$$

This concept has been generalized for set-valued affine functions in different ways (see, e.g. Deutsch and Singer [5], Nikodem and Popa [9], Tan [14], and the references therein). The following definition, inspired from [14], is suitable for the purposes of our paper.

Definition 3.1. A set-valued function $G: X \rightarrow \mathcal{P}(Y)$ is said to be affine if

$$
G\left((1-t) x^{1}+t x^{2}\right)=(1-t) G\left(x^{1}\right)+t G\left(x^{2}\right)
$$

for all $x_{1}, x_{2} \in \operatorname{dom} G$ and $t \in \mathbb{R}$.

Remark 3.2. It can be easily seen that if $G: X \rightarrow \mathcal{P}(Y)$ is a set-valued affine function, then $\operatorname{dom} G$ is affine, since for any $x_{1}, x_{2} \in \operatorname{dom} G$ and $t \in[0,1]$, the equality (3.1) implies $G\left((1-t) x^{1}+t x^{2}\right) \neq \emptyset$, i.e., $(1-t) x^{1}+t x^{2} \in \operatorname{dom} G$. Moreover, for every $x \in X$, the set $G(x)$ is affine. Indeed, letting $x_{1}=x_{2}=x$ in (3.1) we get

$$
G(x)=G((1-t) x+t x)=(1-t) G(x)+t G(x)
$$

for all $t \in \mathbb{R}$, hence $G(x)$ is affine.

Example 3.3. Let $g: E \rightarrow Y$ be a vector-valued affine function, defined on a nonempty affine set $E \subseteq X$. In view of Remark 2.4, we can identify $g$ with the set-valued function $G: X \rightarrow \mathcal{P}(Y)$, given by

$$
G(x)=\left\{\begin{array}{cl}
\{g(x)\} & \text { if } x \in E \\
\emptyset & \text { if } x \in X \backslash E .
\end{array}\right.
$$

It is easy to check that $G$ is affine.

Proposition 3.4. For any set-valued function $G: X \rightarrow \mathcal{P}(Y)$ the following assertions are equivalent:

$1^{\circ} G$ is affine.

$2^{\circ}$ For all $x^{1}, x^{2} \in \operatorname{dom} G$ and $t \in \mathbb{R}$ we have

$$
(1-t) G\left(x^{1}\right)+t G\left(x^{2}\right) \subseteq G\left((1-t) x^{1}+t x^{2}\right) .
$$


Proof. The implication $1^{\circ} \Longrightarrow 2^{\circ}$ is obvious, while $2^{\circ} \Longrightarrow 1^{\circ}$ can be seen as a particular instance of a known result by Nikodem and Popa [9, Prop. 2.11], applied to the restriction of $G$ to $E:=\operatorname{dom} G$, which is an affine set in view of Remark 3.2.

Remark 3.5. In the paper by Tan [14] the set-valued affine functions are defined on a nonempty affine set $E \subseteq X$ taking values in $\mathcal{P}_{0}(Y):=\mathcal{P}(Y) \backslash\{\emptyset\}$. Actually, if $G: X \rightarrow \mathcal{P}(Y)$ is a proper set-valued affine function, then the set $E:=\operatorname{dom} G$ is nonempty and affine, therefore the restriction of $G$ to $E$, i.e., $\left.G\right|_{\operatorname{dom} G}: E \rightarrow \mathcal{P}_{0}(Y)$, is affine in the sense of Tan.

Theorem 3.6. Let $G: X \rightarrow \mathcal{P}(Y)$ be a set-valued affine function. Then the inverse of $G$, i.e., the set-valued function $G^{-1}: Y \rightarrow \mathcal{P}(X)$, is affine.

Proof. According to Proposition 3.4, we just need to show that for any $y_{1}, y_{2} \in$ $\operatorname{dom} G^{-1}=G(X)$ and $t \in \mathbb{R}$ the following inclusion holds:

$$
(1-t) G^{-1}\left(y_{1}\right)+t G^{-1}\left(y_{2}\right) \subseteq G^{-1}\left((1-t) y_{1}+t y_{2}\right)
$$

To this aim, let $x \in(1-t) G^{-1}\left(y_{1}\right)+t G^{-1}\left(y_{2}\right)$. Then there exist $x^{1} \in G^{-1}\left(y_{1}\right)$ and $x^{2} \in G^{-1}\left(y_{1}\right)$ such that $x=(1-t) x^{1}+t x^{2}$. In view of $(2.4)$, we have $x^{1}, x^{2} \in \operatorname{dom} G$. Taking into account that function $G$ is affine, we deduce that

$$
(1-t) y^{1}+t y^{2} \in(1-t) G\left(x_{1}\right)+t G\left(x_{2}\right)=G\left((1-t) x^{1}+t x^{2}\right)=G(x),
$$

which entails $x \in G^{-1}\left((1-t) y^{1}+t y^{2}\right)$. Thus (3.2) holds.

Corollary 3.7. If $g: E \rightarrow Y$ is a vector-valued affine function, defined on a nonempty affine set $E \subseteq X$, then the set-valued function $g^{-1}: Y \rightarrow \mathcal{P}(X)$ is affine.

Proof. Follows by Theorem 3.6, in view of Remark 2.4.

The following two results are based on Tan [14, Props. 4 and 5].

Proposition 3.8. If $G: X \rightarrow \mathcal{P}(Y)$ is a proper set-valued affine function, then there is a unique linear subspace $M \subseteq Y$ such that

$$
G(x)=y+M
$$

for all $x \in \operatorname{dom} G$ and $y \in G(x)$.

Proposition 3.9. If $G: X \rightarrow \mathcal{P}(Y)$ is a proper set-valued affine function, then $G$ possesses an affine selection, i.e., there exists a vector-valued affine function $g: \operatorname{dom} G \rightarrow Y$ such that, for all $x \in \operatorname{dom} G$,

$$
g(x) \in G(x) .
$$

Corollary 3.10. If $G: X \rightarrow \mathcal{P}(Y)$ is a proper set-valued affine function, then there exist a vector-valued affine function $g: \operatorname{dom} G \rightarrow Y$ and a linear subspace $M \subseteq Y$ such that, for all $x \in \operatorname{dom} G$,

$$
G(x)=g(x)+M .
$$

Proof. It is a straightforward consequence of Propositions 3.8 and 3.9. 
Remark 3.11. If $Y=\mathbb{R}$, then there are only two types of proper set-valued affine functions $G: \mathbb{R} \rightarrow \mathcal{P}(\mathbb{R})$, namely: (i) $G(x)=\{g(x)\}$ for all $x \in \operatorname{dom} G$, where $g: \operatorname{dom} G \rightarrow \mathbb{R}$ is an affine function, or (ii) $G(x)=\mathbb{R}$ for all $x \in \operatorname{dom} G$. In particular, when $X=\mathbb{R}$ the domain $\operatorname{dom} G$ is either a singleton or the entire $\mathbb{R}$, in view of Remark 3.2.

\section{Ratios of affine functions}

\subsection{Vector-valued ratios of affine functions}

We begin this section by extending the notion of vector-valued ratios of affine functions, originally introduced by Rothblum [11] within finite-dimensional Euclidean spaces, to the framework of general real linear spaces.

Definition 4.1. A vector-valued function $f: D \rightarrow Y$, defined on a nonempty convex set $D \subseteq X$, is said to be a ratio of affine functions if there exist a vector-valued affine function $g: X \rightarrow Y$ and a real-valued affine function $h: X \rightarrow \mathbb{R}$, such that

$$
D \subseteq\{x \in X \mid h(x)>0\}
$$

and

$$
f(x)=\frac{g(x)}{h(x)}, \forall x \in D .
$$

Remark 4.2. Since $D$ is assumed to be nonempty in Definition 4.1, it is understood that the set $\{x \in X \mid h(x)>0\}$ is nonempty.

The following propositions extend to the framework of general real linear spaces some results obtained within $\mathbb{R}^{n}$ by Rothblum (see [11, Props. 1, 2 and 3] along with subsequent remarks). Their proofs are omitted, since they follow the main lines in [11].

Proposition 4.3. Given a vector-valued function $f: D \rightarrow Y$ defined on a nonempty convex set $D \subseteq X$, the following assertions are equivalent:

$1^{\circ} \operatorname{conv} f(S) \subseteq f(\operatorname{conv} S)$ for every set $S \subseteq D$.

$2^{\circ} f(A)$ is convex for every convex set $A \subseteq D$, i.e., $f$ preserves the convexity of sets by direct images.

Proposition 4.4. Given a vector-valued function $f: D \rightarrow Y$ defined on a nonempty convex set $D \subseteq X$, the following assertions are equivalent:

$1^{\circ} f(\operatorname{conv} S) \subseteq \operatorname{conv} f(S)$ for every set $S \subseteq D$.

$2^{\circ} f^{-1}(B)$ is convex for every convex set $B \subseteq Y$, i.e., function $f$ preserves the convexity of sets by inverse images.

Proposition 4.5. Let $D \subseteq X$ be a nonempty convex set. If $f: D \rightarrow Y$ is a vector-valued ratio of affine functions, then

$$
\operatorname{conv} f(S)=f(\operatorname{conv} S) \text { for every set } S \subseteq D .
$$

Therefore $f$ preserves the convexity of sets by direct and inverse images. 


\subsection{Set-valued ratios of affine functions}

In this section we introduce a class of set-valued ratios of affine functions, by slightly modifying the one proposed by us in [10, Def. 3.3].

Definition 4.6. Let $F: X \rightarrow \mathcal{P}(Y)$ be a set-valued function, whose domain $\operatorname{dom} F=$ : $D \subseteq X$ is a nonempty convex set. We say that $F$ is a ratio of affine functions if there exist a proper set-valued affine function $G: X \rightarrow \mathcal{P}(Y)$ and a real-valued affine function $h: X \rightarrow \mathbb{R}$, such that

$$
D \subseteq\{x \in X \mid h(x)>0\} \cap \operatorname{dom} G
$$

and

$$
F(x)=\left\{\begin{array}{cl}
\frac{G(x)}{h(x)} & \text { if } x \in D \\
\emptyset & \text { if } x \in X \backslash D .
\end{array}\right.
$$

Remark 4.7. As we have pointed out in Remark 4.2, since $D$ is nonempty, the set $\{x \in X \mid h(x)>0\}$ is nonempty as well. In particular, we deduce that any proper set-valued affine function $F: X \rightarrow \mathcal{P}(Y)$ is a ratio of affine functions of type (4.2). Indeed, in this case, $D:=\operatorname{dom} F$ is a nonempty affine hence convex set, $G(x)=F(x)$ and $h(x)=1$ for all $x \in X$.

Theorem 4.8. Let $F: X \rightarrow \mathcal{P}(Y)$ be a set-valued ratio of affine functions defined by (4.2). Then there exist a vector-valued ratio of affine functions $f: D \rightarrow Y$ and $a$ linear subspace $M \subseteq Y$, such that

$$
F(x)=\left\{\begin{array}{cl}
f(x)+M & \text { if } x \in D \\
\emptyset & \text { if } x \in X \backslash D .
\end{array}\right.
$$

Proof. In view of Corollary 3.10, we can find a vector-valued affine function $g: X \rightarrow$ $Y$ and a linear subspace $M \subseteq Y$, such that $G(x)=g(x)+M$ for any $x \in D$. Consequently, we get

$$
F(x)=\frac{G(x)}{h(x)}=\frac{g(x)+M}{h(x)}=\frac{g(x)}{h(x)}+\frac{M}{h(x)}=\frac{g(x)}{h(x)}+M, \forall x \in D .
$$

Thus, we can define a vector-valued ratio of affine functions, $f: D \rightarrow Y$, by

$$
f(x):=\frac{g(x)}{h(x)}, \forall x \in D
$$

which satisfies (4.3).

The following result is a set-valued counterpart of Proposition 4.5 and recovers a similar result obtained in [10, Th. 3.1].

Corollary 4.9. Let $F: X \rightarrow \mathcal{P}(Y)$ be a set-valued ratio of affine functions defined by (4.2). Then, for any set $S \subseteq D$ we have

$$
\operatorname{conv} F(S)=F(\operatorname{conv} S) \text {. }
$$


Proof. According to Theorem 4.8, there exist a vector-valued ratio of affine functions $f: D \rightarrow Y$ and a linear subspace $M \subseteq Y$ such that $F$ has the form (4.3). Consider an arbitrary set $S \subseteq D$. On the one hand, we have $F(S)=f(S)+M$, hence

$$
\operatorname{conv} F(S)=\operatorname{conv}(f(S)+M) \text {. }
$$

On the other hand, we have $F(\operatorname{conv} S)=f(\operatorname{conv} S)+M$, which in view of Proposition 4.5 means

$$
F(\operatorname{conv} S)=\operatorname{conv} f(S)+M .
$$

Taking into account that $M$ is convex, it is a simple exercise to check that

$$
\operatorname{conv} f(S)+M=\operatorname{conv}(f(S)+M) \text {. }
$$

Thus, (4.4) follows by (4.5), (4.6) and (4.7).

In what follows we will show that, similarly to vector-valued ratios of affine functions, the set-valued ratios of affine functions preserve the convexity of sets by direct and inverse images. To this aim we establish two preliminary results for more general classes of set-valued functions.

Theorem 4.10. Consider a set-valued function $F: X \rightarrow \mathcal{P}(Y)$ of type (4.3), where $D \subseteq X$ and $M \subseteq Y$ are nonempty convex sets, while $f: D \rightarrow Y$ is a vector-valued function that preserves the convexity of sets by direct images. Then, for every convex set $A \subseteq X$, the set $F(A)$ is convex, i.e., $F$ preserves the convexity of sets by direct images.

Proof. Let $A \subseteq X$ be a convex set. Since $\operatorname{dom} F=D$, we have

$$
F(A)=F(A \cap D) \cup F(A \backslash D)=F(A \cap D)=f(A \cap D)+M .
$$

By hypothesis, $f(A \cap D)$ is convex as being the image of the convex set $A \cap D$ by $f$. Since $M$ is convex too, we conclude that $F(A)$ is convex.

Theorem 4.11. Consider a set-valued function $F: X \rightarrow \mathcal{P}(Y)$ of type (4.3), where $D \subseteq X$ and $M \subseteq Y$ are nonempty convex sets, while $f: D \rightarrow Y$ is a vector-valued function that preserves the convexity of sets by inverse images. Then, for every convex set $B \subseteq Y$, the sets $F^{-1}(B)$ and $F^{+1}(B) \cap \operatorname{dom} F$ are convex, i.e., $F$ preserves the convexity of sets by lower inverse images as well as by upper inverse images in the sense of Berge.

Proof. First notice that $\operatorname{dom} F=D$. For every convex set $B \subseteq Y$ we have

$$
\begin{aligned}
F^{-1}(B) & =\{x \in X \mid F(x) \cap B \neq \emptyset\} \\
& =\{x \in D \mid(f(x)+M) \cap B \neq \emptyset\} \\
& =\bigcup_{m \in M}\{x \in D \mid f(x)+m \in B\} \\
& =\bigcup_{m \in M} f^{-1}(B-m) \\
& =f^{-1}(B-M) .
\end{aligned}
$$


Since $B-M$ is convex (by convexity of $B$ and $M$ ), its inverse image by $f$, i.e., $f^{-1}(B-M)$, is convex as well. Consequently, the set $F^{-1}(B)$ is convex.

In order to prove that $F^{+1}(B) \cap \operatorname{dom} F$, i.e., $F^{+1}(B) \cap D$, is convex, let

$$
x^{1}, x^{2} \in F^{+1}(B) \cap D \text {. }
$$

We have to show that

$$
\operatorname{conv}\left\{x^{1}, x^{2}\right\} \subseteq F^{+1}(B) \cap D .
$$

Since $D$ is convex set, we just have to check that conv $\left\{x^{1}, x^{2}\right\} \subseteq F^{+1}(B)$. In view of Remark 2.2 , this actually means that $F\left(\operatorname{conv}\left\{x^{1}, x^{2}\right\}\right) \subseteq B$, which by (4.3) reduces to

$$
f\left(\operatorname{conv}\left\{x^{1}, x^{2}\right\}\right)+M \subseteq B .
$$

Observe that, by applying Proposition 4.4 for $S:=\left\{x^{1}, x^{2}\right\}$, we have

$$
\begin{aligned}
f\left(\operatorname{conv}\left\{x^{1}, x^{2}\right\}\right)+M & \subseteq \operatorname{conv} f\left(\left\{x^{1}, x^{2}\right\}\right)+M \\
& =\operatorname{conv}\left\{f\left(x^{1}\right), f\left(x^{2}\right)\right\}+M .
\end{aligned}
$$

Taking into account that $M$ is convex, we can deduce that

$$
\begin{aligned}
\operatorname{conv}\left\{f\left(x^{1}\right), f\left(x^{2}\right)\right\}+M & =\operatorname{conv}\left(\left(f\left(x^{1}\right)+M\right) \cup\left(f\left(x^{2}\right)+M\right)\right) \\
& =\operatorname{conv}\left(F\left(x^{1}\right) \cup F\left(x^{2}\right)\right) .
\end{aligned}
$$

Finally, recalling that $x^{1}, x^{2} \in F^{+1}(B)$, we have $F\left(x^{1}\right) \cup F\left(x^{2}\right) \subseteq B$, which by convexity of $B$ yields

$$
\operatorname{conv}\left(F\left(x^{1}\right) \cup F\left(x^{2}\right)\right) \subseteq B .
$$

Hence, the desired inclusion (4.8) holds.

As a direct consequence of Theorems 4.8, 4.10 and 4.11 we obtain the following result.

Corollary 4.12. If $F: X \rightarrow \mathcal{P}(Y)$ is a set-valued ratio of affine functions defined by (4.2), then the following assertions hold:

$1^{\circ} F$ preserves the convexity of sets by direct images.

$2^{\circ} F$ preserves the convexity of sets by lower inverse images.

$3^{\circ} F$ preserves the convexity of sets by upper inverse images in the sense of Berge.

Remark 4.13. Corollary $4.12\left(1^{\circ}\right)$ extends a result obtained in [10, Cor. 3.1].

Remark 4.14. Assertions $2^{\circ}$ and $3^{\circ}$ of Corollary 4.12, can be interpreted in terms of generalized convexity. They actually show that the restriction $\left.F\right|_{\operatorname{dom} F}: D \rightarrow$ $\mathcal{P}_{0}(Y)$ of any set-valued ratio of affine functions defined by (4.2) is quasiconvex and quasiconcave in the sense of Nikodem [8, Th. 7.2]. Assertion $3^{\circ}$ also means that $F$ is (u1)-type $\left\{0_{Y}\right\}$-quasiconvex in the sense of Seto, Kuroiwa and Popovici [12, Def. 3.3], where $0_{Y}$ stands for the origin of $Y$. Actually, the more general set-valued functions involved in Theorem 4.11 also satisfy these generalized convexity properties as well.

Remark 4.15. In contrast to Corollary $4.12\left(3^{\circ}\right)$, if $F: X \rightarrow \mathcal{P}(Y)$ is a set-valued (even single-valued) ratio of affine functions defined by (4.2), the upper inverse image $F^{+1}(B)$ of a convex set $B \subseteq Y$ in the sense of Aubin-Frankowska is not necessarily convex, as the following example shows. 
Example 4.16. Let $X=Y=\mathbb{R}$ and $D=[0,1]$. Let $F: \mathbb{R} \rightarrow \mathcal{P}(\mathbb{R})$ be the set-valued function defined as

$$
F(x)=\left\{\begin{array}{cl}
\{x\} & \text { if } x \in[0,1] \\
\emptyset & \text { if } x \notin[0,1] .
\end{array}\right.
$$

In view of Remarks 3.11 and $4.7, F$ is a set-valued ratio of affine functions of type (4.2), where $G(x)=\{x\}$ and $h(x)=1$ for all $x \in \mathbb{R}$. Consider the convex set $B=\{0\}$. Although $F^{+1}(B) \cap \operatorname{dom} F=\{0\}$ is convex, the set $\left.\left.F^{+1}(B)=\mathbb{R} \backslash\right] 0,1\right]$ is not convex.

Remark 4.17. Let $F: X \rightarrow \mathcal{P}(Y)$ be a proper set-valued affine function, whose domain is the affine set $D:=\operatorname{dom} F$. Notice that $F$ is a set-valued ratio of affine functions, in view of Remark 4.7. Then, for every convex set $B \subseteq Y$, the sets

$$
F^{-1}(B) \text { and } F^{+1}(B) \cap D
$$

are convex, according to Corollary $4.12\left(2^{\circ}\right.$ and $\left.3^{\circ}\right)$.

On the other hand, by Theorem 3.6, the inverse of $F$, i.e., the set-valued function $F^{-1}: Y \rightarrow \mathcal{P}(X)$, is also affine with $\operatorname{dom} F^{-1}=F(X)=F(D)$. Thus, by applying the above arguments to $F^{-1}$ in the role of $F$, we deduce that for any convex set $A \subseteq X$ the sets

$$
\left(F^{-1}\right)^{-1}(A) \text { and }\left(F^{-1}\right)^{+1}(A) \cap F(D)
$$

are convex. Of course, the convexity of $\left(F^{-1}\right)^{-1}(A)$ is simply recovered by Corollary $4.12\left(1^{\circ}\right)$ applied to $F^{-1}$ in the role of $F$, since $\left(F^{-1}\right)^{-1}(A)=F(A)$. However, the convexity of $\left(F^{-1}\right)^{+1}(A) \cap F(D)$ is not a simple consequence of the convexity-preserving properties of $F$. Indeed, by applying (2.3) for $F^{-1}$ in the role of $F$, we get

$$
\left(F^{-1}\right)^{+1}(A)=Y \backslash F(X \backslash A)=Y \backslash F(D \backslash A),
$$

hence

$$
\begin{aligned}
\left(F^{-1}\right)^{+1}(A) \cap F(D) & =(Y \backslash F(D \backslash A)) \cap F(D) \\
& =F(D) \backslash F(D \backslash A) .
\end{aligned}
$$

Notice that $F(D) \backslash F(D \backslash A) \subseteq F(D \cap A)$ and $F(D \cap A)$ is convex, according to Corollary $4.12\left(1^{\circ}\right)$, since $D \cap A$ is convex. However, $F(D) \backslash F(D \backslash A) \neq F(D \cap A)$ in general, as for instance when $F: \mathbb{R} \rightarrow \mathcal{P}(\mathbb{R})$ is the constant ratio of affine functions defined by $F(x)=\{1\}$ for all $x \in \mathbb{R}$ and $A=\{0\} \subseteq D=\mathbb{R}$.

\section{Conclusions}

Theorem 4.8 gives a useful characterization of the set-valued ratios of affine functions, as reflected in its Corollaries 4.9 and 4.9. Actually, the special structure of these functions, given by Theorem 4.8, suggested us to consider in Theorems 4.10 and 4.11 some broader classes of set-valued functions that enjoy the convexity-preserving properties of the set-valued ratios of affine functions pointed out in Corollary 4.9. An interesting question arises, namely whether it would be possible to extend these classes by replacing the convex set $M$ in Theorems 4.10 and 4.11 (which can be seen as a constant affine set-valued function) by appropriate set-valued functions. In general, this is not true even if $M$ is replaced by a single-valued function that 
preserves the convexity of sets by direct and inverse images. For instance, consider $D=[0,1] \subseteq X=\mathbb{R}, Y=\mathbb{R}^{2}$ and let $F: \mathbb{R} \rightarrow \mathcal{P}\left(\mathbb{R}^{2}\right)$ be the set-valued function defined as

$$
F(x)=\left\{\begin{array}{cl}
f(x)+M(x) & \text { if } x \in[0,1] \\
\emptyset & \text { if } x \notin[0,1]
\end{array}\right.
$$

where $f:[0,1] \rightarrow \mathbb{R}^{2}$ is a vector-valued ratio of affine functions given by

$$
f(x)=\frac{g(x)}{h(x)}=\frac{(1,1)}{x+1} \quad \text { for all } x \in[0,1]
$$

and $M: \mathbb{R} \rightarrow \mathcal{P}\left(\mathbb{R}^{2}\right)$ is a single-valued affine function, given by

$$
M(x)=\{(x,-x)\} \quad \text { for all } x \in \mathbb{R} .
$$

Consider the convex sets $A=[0,1]$ and $B=\operatorname{conv} F(\{0,1\})$. It is easy to check that $B$ is a line segment with end points $(1,1)$ and $(3 / 2,-1 / 2)$, while $F(A)$ is an arc of hyperbola joining these points. On the other hand, we have $F^{-1}(B)=\{0,1\}$ and $\left.F^{+1}(B)=\mathbb{R} \backslash\right] 0,1\left[\right.$. Obviously, the sets $F(A), F^{-1}(B)$ and $F^{+1}(B)$ are not convex. It is worth to mention that similar examples, where $F$ preserves the convexity of sets by direct and inverse images, can be given by considering ratios af affine functions $f=\frac{g}{h}$ and $M=\frac{G}{h}$ with the same denominator. However, such a configuration always leads to the trivial case where $F$ itself is a ratio of affine functions satisfying the properties in demand by virtue of Corollary 4.9 .

\section{References}

[1] Aubin, J.-P., Frankowska, H., Set-Valued Analysis, Birhäuser, Boston, 1990.

[2] Avriel, M., Diewert, W.E., Schaible S., Zang, I., Generalized Concavity, Plenum Press, New York, 1988.

[3] Berge, C., Topological Spaces Including a Treatment of Multi-Valued Functions, Vector Spaces and Convexity, Oliver \& Boyd, Edinburgh-London, 1963.

[4] Cambini, A., Martein, L., Generalized Convexity and Optimization: Theory and Applications, Springer-Verlag, Berlin, 2009.

[5] Deutsch, F., Singer, I., On single-valuedness of convex set-valued maps, Set-Valued Anal., 1(1993), 97-103.

[6] Göpfert, A., Riahi, H., Tammer, C., Zălinescu, C., Variational Methods in Partially Ordered Spaces, Springer-Verlag, New York, 2003.

[7] Kuroiwa, D., Popovici, N., Rocca, M., A characterization of cone-convexity for set-valued functions by cone-quasiconvexity, Set-Valued Var. Anal., 23(2015), 295-304.

[8] Nikodem, K., K-Convex and K-Concave Set-Valued Functions, Habilitation Dissertation, Scientific Bulletin of Łódź Technical University, nr. 559, 1989.

[9] Nikodem, K., Popa, D., On single-valuedness of set-valued maps satisfying linear inclusions, Banach J. Math. Anal., 3(2009), 44-51.

[10] Orzan, A., A new class of fractional type set-valued functions, Carpathian J. Math., 35(2019), 79-84. 
[11] Rothblum, U.G., Ratios of affine functions, Math. Program., 32(1985), 357-365.

[12] Seto, K., Kuroiwa, D., Popovici, N., A systematization of convexity and quasiconvexity concepts for set-valued maps, defined by l-type and u-type preorder relations, Optimization, 67(2018), 1077-1094.

[13] Stancu-Minasian, I.M., Fractional Programming. Theory, Methods and Applications, Mathematics and its Applications, Kluwer-Dordrecht 409, 1997.

[14] Tan, D.H., A note on multivalued affine mappings, Stud. Univ. Babeş-Bolyai Math., 33(1988), 55-59.

\author{
Alexandru Orzan \\ Babeş-Bolyai University, \\ Faculty of Mathematics and Computer Sciences, \\ 1, Kogălniceanu Street, \\ 400084 Cluj-Napoca, Romania \\ e-mail: alexandru.orzan@ubbcluj.ro \\ Nicolae Popovici \\ Babeş-Bolyai University, \\ Faculty of Mathematics and Computer Sciences, \\ 1, Kogălniceanu Street, \\ 400084 Cluj-Napoca, Romania \\ e-mail: popovici@math.ubbcluj.ro
}

